\title{
Les difficultés d'adaptation psychosociale et les aptitudes intellectuelles chez les élèves à risque d'échec scolaire
}

\author{
Mohammed ECHARGHAOUI ${ }^{{ }^{*}}$ \\ ${ }^{a}$ Université Mohammed V, Rabat, Maroc \\ * echarghaoui@gmail.com
}

\section{Résumé}

Cette étude vise à déterminer les difficultés d'adaptation psychosociale, d'identifier les aptitudes intellectuelles chez les élèves à risque d'échec scolaire et de chercher profondément les facteurs d'adaptation psychosociale ayant un pouvoir discriminatif. L'échantillon se formait de certains élèves au niveau secondaire, présentant des risques d'échec scolaire. On s'est servi de six subtests de la Batterie NV7. A l'issue d'un ensemble d'analyses, on a constaté que la majorité des élèves à risque d'échec scolaire présente de moyennes aptitudes intellectuelles qui diffèrent selon le sexe et le niveau scolaire. Ces derniers ont des difficultés psychosociales au niveau du sentiment de compétence, de la volonté d'apprendre en français, de l'avantage à décrocher, du climat éducatif et de l'estime de soi. La volonté d'apprendre, le sentiment de compétence en français \& en mathématiques, et l'attrait envers l'école influencent leur adaptation.

Mots clés : Adaptation psychosociale, Echec scolaire, Intelligence, NV7.

\section{Psychosocial adjustment difficulties and intellectual skills in students at risk of school failure}

\begin{abstract}
This study aims to determine the difficulties of psychosocial adaptation, identify the intellectual abilities among students at risk of school failure, and scrutinize discriminatory variables among psychosocial adjustment factors. The sample is
\end{abstract}


some high school students who were at risk of school failure. We used six subtests of the NV7 Battery. At the end of a set of analyses, we have found that the majority of students at risk of school failure have average intellectual abilities which differ by gender and grade level. They have psychosocial difficulties on the level of the emotional competence, the will to learn using French, the benefits to be gained, the educational climate and self-esteem. The will to learn, the emotional competence in French and mathematics, and the attraction to school influence their adaptation.

Keywords : psychosocial adaptation, school failure, intelligence, NV7.

\section{Introduction et problématique}

De nos jours, la question de l'échec scolaire devient un sujet social par excellence, de plus en plus préoccupant au sein du secteur de l'éducation. Plusieurs chercheurs, notamment les psychologues, les pédagogues, les sociologues et les économistes, ont conçu un ensemble d'études où les angles d'observation diffèrent l'un de l'autre.

Les recherches ont abordé ce problème dans son rapport avec des facteurs liés à l'école, entre autres le système de notation, le système de dévalorisation en relation avec le phénomène de redoublement ou encore dans son rapport avec les problèmes sociaux et familiaux. D'autres recherches ont mis l'accent sur les problèmes linguistiques. Ce constat nous a permis de comprendre que l'échec scolaire se traduit par un redoublement, un retard scolaire, un abandon scolaire, sans achever le cycle scolaire (Francine, 1997) ou encore traduit par de mauvaises notes (Gimeno, 1984). Aussi existent-ils d'autres indicateurs de l'échec scolaire, notamment : le taux de redoublement, l'entrée précoce au lycée professionnel, l'orientation vers l'éducation spécialisée, etc. (Weil-Barais, 2001).

Le système éducatif Marocain comme celui de plusieurs pays en voie de développement, est secoué par une crise à propos de l'échec scolaire, phénomène qui ne fait que s'aggraver. L'apparition de la charte nationale en 1999 décline jusqu'à nos 
jours un ensemble de questions majeures du système de l'éducation nationale dont la nature freine les réformes, en particulier celle citée précédemment.

Plus particulièrement au sein de notre contexte social, on n'accorde pas de l'importance à l'identification des difficultés psychosociales et aux aptitudes intellectuelles, des élèves à risque d'échec scolaire. La plupart des études déjà conçues dans ce domaine traitent souvent les facteurs responsables qui conduisent à ce problème. D'ailleurs, les exigences en qualifications deviennent de plus en plus primordiales. Il est évident que l'apprentissage diffère d'une personne à une autre, alors que la difficulté réside dans la manière d'apprendre : seuls quelques élèves sont retenus par l'école du moment que cette dernière ne peut pas s'adapter à tout le monde.

Quoique les formes de l'échec scolaire soient bien caractérisées, ce dernier demeure une réalité très complexe. L'échec scolaire est pensé en termes de défaillance, de manque, de privation ; l'individu est en échec par défaut d'intelligence, par manque de volonté ou par inhibition de ses capacités intellectuelles. Aux dires de Mireille Cifali (1994), « être en échec » est relatif à «être épinglé en négatif : l'élève ne sait pas, ne peut pas, n'arrive pas, et cette description en négatif entraine une volonté de transformer, redresser, d'extirper, et de remplacer l'insuffisance par la qualité » (Yanni, 2001).

C'est dans cette perspective que cette étude a été conçu. Il s'agit de déterminer les difficultés psychosociales, d'évaluer et d'analyser les aptitudes intellectuelles chez les élèves à risque d'échec scolaire au niveau secondaire qualifiant pour établir un pronostic de réussite, en vue d'aboutir à un modèle préventif propre aux élèves à risque d'échec scolaire. Ce dernier vise à prévoir et prédire l'adaptation d'un sujet au cours de son cursus scolaire, et plus tard universitaire. 
Ceci nous conduit à poser la question suivante : La situation des élèves à risque d'échec scolaire dépend-elle plus des difficultés psychosociales que des aptitudes intellectuelles?

\section{Participants}

Pour identifier les élèves à risque d'échec scolaire du niveau secondaire qui présentent des difficultés, on s'est servi de l'ensemble des dossiers des élèves appropriés à chacun d'eux. Ces dossiers comprennent l'ensemble des bulletins, le rapport médical, les fiches d'absence, outre que celles de réorientation. Les élèves qui étudient au tronc commun science et professionnel sont au nombre de 142 élèves (soit 36,98\%), en $1^{\text {ère }}$ année bacscience expérimentale au nombre de 130 élèves (soit 33,85\%), ainsi que ceux en $2^{\text {ème }}$ année bac-science expérimentale au nombre de 112 élèves (soit 29,17\%) à la direction de Rabat, Maroc.

Par conséquent, et à travers l'étude de l'ensemble des cas (nombre total=384 élèves $)^{1}$, nous avons abouti à dégager un nombre défini des élèves qui peuvent subvenir aux exigences de cette étude. Ce dernier vaut 76 élèves.

L'échantillon est constitué de 76 élèves qui fréquentent l'école secondaire publique. Cet échantillon permet de recueillir des données auprès de 64 sujets avec un taux de réponses égales $86,5 \%$. L'échantillon se compose de 20 sujets en tronc commun science et professionnel $(15 \mathrm{M} ; 5 \mathrm{~F}), 18$ sujets en $1^{\text {ère }}$ baccalauréat $(11 \mathrm{M}, 7 \mathrm{~F})$ et 26 sujets $(9 \mathrm{M} ; 17 \mathrm{~F})$ en $2^{\text {ème }}$ baccalauréat.

1- NB : Afin de faciliter la tâche, la sélection a fait intervenir quelques membres du cadre pédagogique, qui sont au courant de la situation de la majorité des sujets à propos de leurs études, absence, orientation. 207 


\section{Mesures}

On s'est servi de deux instruments de mesure : La batterie NV7 comprend dix subtests., nous avons utilisé les subtests suivants : Raisonnement déductif (R1): 24 items (1; 2; $3 ; \ldots ; 24)$; Raisonnement inductif (R2): 29 items $(25 ; 26 ; 27 ; \ldots ; 53)$; Problèmes (P) : 16 items $(205 ; 206 ; 207 ; \ldots ; 220)$; Attention (A) : 55 items $(301 ; 302 ; \ldots ; 355)$; Compréhension verbale (CV): 55 items (356; $357 ; 358 ; \ldots ; 410)$; Orthographe (O): 55 items $(411 ; 412 ; 413 ; \ldots ; 465)$.

\section{La sensibilité}

Tableau 1. Moyennes et écart-types des notes brutes pour les différents subtests

\begin{tabular}{llllll}
\hline Intitulé du subtest & $\mathbf{N}$. items & N. sujets & Total brut & Moyenne & Écart-type \\
\hline Raisonnement déductif (R1) & 24 & 64 & 24 & 17,3 & 3,07 \\
\hline Raisonnement inductif (R2) & 29 & 64 & 29 & 18,1 & 4,36 \\
\hline Problèmes (P) & 16 & 64 & 16 & 6,6 & 2,81 \\
\hline Attention (A) & 55 & 64 & 55 & 48,0 & 6,13 \\
\hline Orthographe (OR) & 55 & 64 & 55 & 25,6 & 7,23 \\
\hline Compréhension verbale (CV) & 55 & 64 & 55 & 27,5 & 10,80 \\
\hline
\end{tabular}

Les scores bruts obtenus aux six subtests, leurs moyennes observées aussi bien que leurs écart types permettent de constater que : La finesse des épreuves de Raisonnement déductif et Raisonnement inductif est satisfaisante ; c'est-à-dire que les formes gaussiennes des courbes et leur dispersion autour de la moyenne sont convenables. En revanche les autres subtests : Attention, Orthographe et Compréhension verbale présentent une distribution proche de la loi normale. 


\section{La fidélité}

Tableau 2. Indicateurs de consistance interne des subtests de la NV7

\begin{tabular}{llll}
\hline Intitulé du subtest & N. items & N. sujets & Alpha de Cronbach \\
\hline Raisonnement déductif (R1) & 24 & 64 & 0,60 \\
\hline Raisonnement inductif (R2) & 29 & 64 & 0,78 \\
\hline Problèmes (P) & 16 & 64 & 0,72 \\
\hline Attention (A) & 55 & 64 & 0,87 \\
\hline Orthographe (OR) & 55 & 64 & 0,80 \\
\hline Compréhension verbal (CV) & 55 & 64 & 0,91 \\
\hline
\end{tabular}

On remarque que les coefficients $\alpha$ de Cronbach se situent entre 0,60 et 0,91 ; dans l'ensemble, les résultats obtenus, $\alpha$ ont des valeurs également acceptables ; satisfaisantes. Seuls, les subtests : Raisonnement déductif $\alpha=0,60$, Problèmes $\alpha=0,72$ et Raisonnement inductif $\alpha=0,78$ présentent des valeurs un peu satisfaisantes.

Le deuxième instrument est le questionnaire psychosocial qui a été développé par les chercheurs de l'équipe d'évaluation de la SIAA (Stratégie d'Intervention Agir Autrement) - Québec.

Concernant notre étude, nous avons utilisé un ensemble de variables, réunis sous le nom «Questionnaire psychosocial ». On en cite : Attrait envers l'école (4 items de 3 à 6) ; Sentiment de compétence en mathématiques (5 items de 7 à 11); Sentiment de compétence en français (5 items de 12 à 16) ; Volonté d'apprendre en mathématiques (4 
items de 17 à 20); Volonté d'apprendre en français (4 items de 21 à 24); Orientation vers le travail scolaire (3 items de 25 à 27); Avantages à décrocher (14 items de 28à 41) ;

Climat éducatif (7 items de 42 à 48); Estime de soi (10 items de 49 à 58).

\section{La sensibilité}

Tableau 3. Analyse de la symétrie de la distribution des variables de l'adaptation psychosociale
(1)
(2)
(3) (4)
(5)
(6) (7)
(8) (9)

\begin{tabular}{llllllllll}
\hline Moyenne & 5,31 & 4,67 & 4,36 & 5,08 & 4,64 & 5,37 & 3,04 & 2,04 & 2,12 \\
\hline Ecart-type & 1,25 & 0,82 & 0,97 & 1,29 & 1,61 & 1,14 & 0,47 & 0,63 & 0,38 \\
\hline Ration d'asymétrie & $-3,30$ & $-2,96$ & $-0,33$ & $-2,18$ & $-1,31$ & $-2,86$ & $-2,68$ & 1,19 & $-0,27$ \\
\hline
\end{tabular}

D'après le tableau ci-dessus, on ne conclut que les quatre variables, à savoir :

Sentiment de compétence en français, Volonté d'apprendre en français, Climat éducatif et Estime de soi présentent des valeurs de ratios d'asymétrie inférieures à 2 en valeur absolue. Ainsi, on peut constater que les données accueillies respectent une distribution normale ; elles sont riches et de qualité.

\section{La fidélité}

Tableau 4. Indicateurs de consistance interne des variables de l'adaptation psychosociale

\begin{tabular}{llll}
\hline Variables d'adaptation psychosociale & N. items & N. sujets & Alpha de Cronbach \\
\hline $\begin{array}{l}\text { Attrait envers l'école (1) } \\
\text { Sentiment de compétence en }\end{array}$ & 4 & 64 & 0,76 \\
\begin{tabular}{l} 
mathématiques (2) \\
\hline
\end{tabular}
\end{tabular}




\begin{tabular}{lccc}
\hline $\begin{array}{l}\text { Sentiment de compétence en français } \\
(3)\end{array}$ & 5 & 64 & 0,57 \\
\hline $\begin{array}{l}\text { Volonté d'apprendre en } \\
\text { mathématiques (4) }\end{array}$ & 4 & 64 & 0,81 \\
\hline $\begin{array}{l}\text { Volonté d'apprendre en français (5) } \\
\text { Orientation vers le travail scolaire (6) }\end{array}$ & 3 & 64 & 0,84 \\
\hline $\begin{array}{l}\text { Avantage à décrocher (7) } \\
\text { Climat éducatif (8) }\end{array}$ & 14 & 64 & 0,47 \\
\hline Estime de soi (9) & 7 & 64 & 0,71 \\
\hline
\end{tabular}

On remarque que les coefficients $\alpha$ de Cronbach se situent entre 0,47 et 0,84 ; dans l'ensemble des résultats obtenus, $\alpha$ ont des valeurs également acceptables ; satisfaisantes.

\section{Résultats}

Tableau 5. Niveau général des aptitudes intellectuelles

\begin{tabular}{|c|c|c|c|c|}
\hline Intitulé du subtest & Niveau d'aptitude & Effectifs & Pourcentage & $\begin{array}{l}\text { Pourcentage } \\
\text { cumulé }\end{array}$ \\
\hline \multirow{3}{*}{$\begin{array}{l}\text { Raisonnement déductif } \\
\text { (R1) }\end{array}$} & Résultats faibles & 16 & 25,0 & 25,0 \\
\hline & Résultats moyens & 31 & 48,4 & 73,4 \\
\hline & Résultats élevés & 17 & 26,6 & 100,0 \\
\hline \multirow{2}{*}{$\begin{array}{l}\text { Raisonnement inductif } \\
\text { (R2) }\end{array}$} & Résultats faibles & 15 & 23,4 & 23,4 \\
\hline & Résultats moyens & 35 & 54,7 & 78,1 \\
\hline
\end{tabular}


The Journal of Quality in Education (JoQiE) Vol.11, N¹7, May 2021

\begin{tabular}{|c|c|c|c|c|}
\hline & Résultats élevés & 14 & 21,9 & 100,0 \\
\hline \multirow[t]{3}{*}{ Problèmes (P) } & Résultats faibles & 14 & 21,9 & 21,9 \\
\hline & Résultats moyens & 30 & 46,9 & 68,8 \\
\hline & Résultats élevés & 20 & 31,3 & 100,0 \\
\hline \multirow[t]{3}{*}{ Attention (A) } & Résultats faibles & 13 & 20,3 & 20,3 \\
\hline & Résultats moyens & 40 & 62,5 & 82,8 \\
\hline & Résultats élevés & 11 & 17,2 & 100,0 \\
\hline \multirow[t]{3}{*}{ Orthographe (OR) } & Résultats faibles & 11 & 17,2 & 17,2 \\
\hline & Résultats moyens & 39 & 60,9 & 78,1 \\
\hline & Résultats élevés & 14 & 21,9 & 100,0 \\
\hline \multirow{3}{*}{$\begin{array}{l}\text { Compréhension verbale } \\
\text { (CV) }\end{array}$} & Résultats faibles & 14 & 21,9 & 21,9 \\
\hline & Résultats moyens & 34 & 53,1 & 75,0 \\
\hline & Résultats élevés & 16 & 25,0 & 100,0 \\
\hline \multirow[t]{3}{*}{ Niveau général du sujet } & Résultats faibles & 10 & 15,6 & 15,6 \\
\hline & Résultats moyens & 49 & 76,6 & 92,2 \\
\hline & Résultats élevés & 5 & 7,8 & 100,0 \\
\hline
\end{tabular}

Le tableau ci-dessus montre que : 17 sujets $(26.6 \%)$ ont un niveau élevé au subtest raisonnement déductif (R1); ce qui indique généralement un bon potentiel de raisonnement. Leurs capacités intellectuelles interviennent dans la compréhension des situations de la vie quotidienne ; c'est-à-dire un bon potentiel d'adaptation à ces situations. En revanche, 31 sujets (48.4\%) ont un niveau moyen qui indique la présence 
de certaines acquisitions de base. Mais, il est limité pour une adaptation quotidienne, par exemple la formation professionnelle. Quant au reste, 16 sujets $(25 \%)$ ont un niveau faible qui indique l'absence d'acquisitions nécessaires outre que la présence des difficultés à opérationnaliser les capacités intellectuelles dans la compréhension des situations de la vie quotidienne.

14 sujets $(21,9 \%)$ ont un niveau élevé au subtest Raisonnement inductif (R2) ; ce qui indique un bon potentiel intellectuel et une vivacité d'esprit, de bonnes capacités de logique, en particulier sur un matériel numérique. Néanmoins, 35 sujets (54.7\%) ont un niveau moyen au subtest (R2); ce qui indique un moyen potentiel intellectuel, une moyenne capacitée de logique. En revanche, 15 (23.4\%) sujets parmi 64 présentent des difficultés au niveau du raisonnement, un manque d'aisance avec les chiffres, lié à des acquisitions scolaires insuffisantes, blocage systématique devant tout matériel numérique, surtout si les résultats sont peu élevés ou faibles au subtest «problèmes $(\mathrm{P}) \gg$.

Pour le subtest « Problèmes », 20 sujets (31.3\%) ont un niveau élevé qui indique un bon potentiel de résolution des problèmes numériques et une bonne intégration des notions de base de mathématiques, signe de réussite scolaire. 30 sujets $(46.9 \%)$ ont un niveau moyen qui indique la présence d'un moyen potentiel de résolution des problèmes en dépit des difficultés au niveau des opérations mathématiques (notions de base en mathématiques). 14 sujets (21.9\%) ont un niveau faible qui indique l'existence des difficultés de calcul, manque de la maitrise des différentes notions mathématiques par exemple, pourcentage, proportion, superficie, etc.

Les résultats obtenus au subtest «Attention » fluctuent entre un niveau faible, moyen et élevé chez les sujets : 11 sujets (17.2\%) ont un niveau élevé, tandis que 40 sujets $(62.5 \%)$ ont un niveau moyen ; ce qui indique que les sujets portent des capacités 
d'attention et de concentration. A cela s'ajoutent 13 sujets $(20,3 \%)$ de niveau faible qui indique un manque de concentration et de motivation dans le travail outre qu'une fatigabilité visuelle.

Pour le subtest orthographe, 14 sujets (21.9\%) ont un niveau élevé ; ils présentent de bonnes acquisitions en orthographe outre qu'une aisance dans l'usage écrit de la langue française. 50 sujets ont obtenu des résultats entre un niveau moyen (60.9\%) et faible (17.2\%); ce qui indique qu'une partie des sujets est moyenne, l'autre est faible au niveau des règles de grammaire et de la langue française.

Pour le subtest Compréhension verbale, les sujets ont obtenu des résultats qui varient entre un niveau faible (21.9\%), moyen $(53.1 \%)$ et élevé $(25 \%)$; ce qui indique une capacité de compréhension du français médiocre pour les sujets à niveau moyen et faible, par contre les autres sujets ont une note élevée qui leur reflète une bonne compréhension verbale, une aisance dans le maniement de la langue française.

Tableau 6. Moyennes et écart-types des notes brutes pour les différents subtests de la batterie NV7 en fonction du sexe

\begin{tabular}{lllll}
\hline & SEXE & $\mathbf{N}$ & $\mathbf{M}$ & ET \\
\hline Raisonnement déductif & $\mathrm{M}$ & 35 & 17,57 & 2,88 \\
\cline { 2 - 5 } & $\mathrm{F}$ & 29 & 17,00 & 3,31 \\
\hline Raisonnement inductif & $\mathrm{M}$ & 35 & 19,26 & 4,40 \\
\cline { 2 - 5 } & $\mathrm{F}$ & 29 & 16,72 & 3,96 \\
\hline Problèmes & $\mathrm{M}$ & 35 & 6,74 & 3,11 \\
\cline { 2 - 5 } & $\mathrm{F}$ & 29 & 6,48 & 2,46 \\
\hline Attention & $\mathrm{M}$ & 35 & 47,34 & 6,93 \\
\hline
\end{tabular}


The Journal of Quality in Education (JoQiE) Vol.11, N¹7, May 2021

\begin{tabular}{lcccc}
\hline & $F$ & 29 & 48,90 & 5,00 \\
\hline Orthographe & $M$ & 35 & 24,11 & 7,61 \\
\cline { 2 - 5 } & $\mathrm{F}$ & 29 & 27,41 & 6,42 \\
\hline Compréhension verbale & $\mathrm{M}$ & 35 & 27,17 & 10,81 \\
\cline { 2 - 5 } & $\mathrm{F}$ & 29 & 27,86 & 10,97 \\
\hline
\end{tabular}

Selon le tableau statistique descriptif précédent, on remarque globalement une différence au niveau des moyennes observées et des écart-types des résultats des six subtests de la batterie NV7des sujet de sexe féminin et masculin. En effet, on observe une petite supériorité des sujets de sexe masculin dans les subtests (R1, R2, P), de même que chez les sujets féminins dans les subtests (A, OR, CV).

Tableau 7. Etude des différences de moyennes dans les subtests de la batterie en fonction du sexe

\begin{tabular}{|c|c|c|c|c|c|c|c|c|c|}
\hline & \multicolumn{2}{|c|}{$\begin{array}{l}\text { Test de } \\
\text { Levene }\end{array}$} & \multicolumn{7}{|c|}{ Test-t pour égalité des moyennes } \\
\hline & \multirow[t]{2}{*}{$\mathrm{F}$} & \multirow[t]{2}{*}{ Sig. } & \multirow[t]{2}{*}{$\mathrm{T}$} & \multirow[t]{2}{*}{ ddl } & \multirow[t]{2}{*}{ Sig. } & \multirow[t]{2}{*}{$\begin{array}{l}\text { Différence } \\
\text { moyenne }\end{array}$} & \multirow[t]{2}{*}{$\begin{array}{l}\text { Différence } \\
\text { écart-type }\end{array}$} & \multicolumn{2}{|c|}{$\begin{array}{l}\text { Intervalle } \\
\text { de } \\
\text { confiance }\end{array}$} \\
\hline & & & & & & & & $\operatorname{lnf}$ & Sup \\
\hline $\begin{array}{l}\text { Raisonnement } \\
\text { déductif }\end{array}$ & 0,63 & 0,43 & 0,74 & 62,00 & 0,46 & 0,57 & 0,77 & $-0,98$ & 2,12 \\
\hline $\begin{array}{l}\text { Raisonnement } \\
\text { inductif }\end{array}$ & 0,13 & 0,73 & 2,40 & 62,00 & 0,02 & 2,53 & 1,06 & 0,42 & 4,65 \\
\hline Problèmes & 1,96 & 0,17 & 0,37 & 62,00 & 0,72 & 0,26 & 0,71 & $-1,16$ & 1,68 \\
\hline
\end{tabular}


The Journal of Quality in Education (JoQiE) Vol.11, N¹7, May 2021

\begin{tabular}{lllllllllll}
\hline Attention & 4,96 & 0,03 & $-1,01$ & 62,00 & 0,32 & $-1,55$ & 1,54 & $-4,63$ & 1,53 \\
\hline $\begin{array}{l}\text { Orthographe } \\
0,30\end{array}$ & 0,59 & $-1,85$ & 62,00 & 0,07 & $-3,30$ & 1,78 & $-6,86$ & 0,26 \\
\hline $\begin{array}{l}\text { Compréhension } \\
\text { verbale }\end{array}$ & 0,02 & 0,88 & $-0,25$ & 62,00 & 0,80 & $-0,69$ & 2,73 & $-6,15$ & 4,77 \\
\end{tabular}

Selon le tableau ci-dessus, le test d'hypothèse Levene montre qu'il n'existe pas de différence significative statistiquement au seuil de signification $\mathrm{p}=0,05$ entre les sujets du sexe masculin et ceux du sexe féminin selon le niveau d'aptitudes intellectuelles dans les différents subtests de la batterie multifactorielle NV7 (R1, R2, P, $\mathrm{OR}, \mathrm{CV}$ ) hormis les résultats du subtest «Attention » qui indiquent une différence significative $(4,96 ; \mathrm{p}=0,03<0,05)$.

0,46 est la probabilité d'obtenir une valeur absolue supérieure ou égale à la statistique t observée $(0,74)$, si la différence entre le groupe masculin et le groupe féminin dans le subtest (R1) est purement due au hasard. Il n'est pas possible de conclure que la différence de moyennes observées n'est pas due au hasard. Donc la vraie différenciation des aptitudes concernant les résultats dans le subtest «Raisonnement déductif » entre les sujets de sexe masculin et féminin ne se trouve pas, au risque d'erreur de 5\%, dans les limites proposées par intervalle de confiance $95 \%$ de la différence.

Tableau 8. Moyennes et écart-type des différents subtests en fonction du niveau scolaire

\begin{tabular}{|c|c|c|c|c|c|c|c|}
\hline & & \multirow[t]{2}{*}{$\mathbf{N}$} & \multirow[t]{2}{*}{ M } & \multirow[t]{2}{*}{ ET } & \multirow[t]{2}{*}{ ES } & \multicolumn{2}{|c|}{ Intervalle de confiance } \\
\hline & & & & & & $\operatorname{lnf}$ & Sup \\
\hline \multirow{2}{*}{$\begin{array}{l}\text { Raisonnement } \\
\text { déductif }\end{array}$} & $\mathrm{TCP} / \mathrm{TCS}$ & 20 & 19,15 & 2,23 & 0,50 & 18,11 & 20,19 \\
\hline & $1^{\text {ère bac }}$ & 18 & 15,83 & 3,79 & 0,89 & 13,95 & 17,72 \\
\hline
\end{tabular}




\begin{tabular}{|c|c|c|c|c|c|c|c|}
\hline & $2^{\text {ème }}$ bac & 26 & 16,92 & 2,40 & 0,47 & 15,95 & 17,89 \\
\hline \multirow{3}{*}{$\begin{array}{l}\text { Raisonnement } \\
\text { inductif }\end{array}$} & TCP/TCS & 20 & 19,00 & 3,96 & 0,89 & 17,15 & 20,85 \\
\hline & $1^{\text {ère }}$ bac & 18 & 16,22 & 4,57 & 1,08 & 13,95 & 18,50 \\
\hline & $2^{\text {ème }}$ bac & 26 & 18,73 & 4,28 & 0,84 & 17,00 & 20,46 \\
\hline \multirow[t]{3}{*}{ Problèmes } & TCP/TCS & 20 & 8,25 & 2,31 & 0,52 & 7,17 & 9,33 \\
\hline & $1^{\text {ère }}$ bac & 18 & 3,61 & 2,68 & 0,63 & 2,28 & 4,94 \\
\hline & $2^{\text {ème }} \mathrm{bac}$ & 26 & 7,46 & 1,27 & 0,25 & 6,95 & 7,98 \\
\hline \multirow[t]{3}{*}{ Attention } & TCP/TCS & 20 & 51,05 & 2,87 & 0,64 & 49,70 & 52,40 \\
\hline & $1^{\text {ère }}$ bac & 18 & 41,17 & 6,72 & 1,58 & 37,82 & 44,51 \\
\hline & $2^{\text {ème }}$ bac & 26 & 50,50 & 3,20 & 0,63 & 49,21 & 51,79 \\
\hline \multirow[t]{3}{*}{ Orthographe } & TCP/TCS & 20 & 23,35 & 6,47 & 1,45 & 20,32 & 26,38 \\
\hline & $1^{\text {ère }}$ bac & 18 & 23,83 & 10,08 & 2,38 & 18,82 & 28,84 \\
\hline & $2^{\text {ème }}$ bac & 26 & 28,58 & 3,92 & 0,77 & 26,99 & 30,16 \\
\hline \multirow{3}{*}{$\begin{array}{l}\text { Compréhension } \\
\text { verbale }\end{array}$} & TCP/TCS & 20 & 29,70 & 8,99 & 2,01 & 25,49 & 33,91 \\
\hline & $1^{\text {ère }}$ bac & 18 & 20,17 & 12,80 & 3,02 & 13,80 & 26,53 \\
\hline & $2^{\text {ème }}$ bac & 26 & 30,85 & 8,19 & 1,61 & 27,54 & 34,15 \\
\hline
\end{tabular}

Une première série d'analyse concernant les subtests des aptitudes intellectuelles selon le niveau scolaire des sujets révèle des différences qui existent pour chacun d'entre les trois niveaux scolaires. Les effets intenses apparaissent au niveau des épreuves «Problèmes » et «Compréhension verbale». 
Les variations les plus importantes sont observées au niveau scolaire : tronc commun science et professionnel. Dans ce cas, les moyennes atteignent un niveau élevé et moins dispersé dans les épreuves : «Raisonnement déductif, Raisonnement inductif, Problèmes et Attention ». Les sujets de la deuxième année baccalauréat ont des moyennes plus élevées dans les épreuves : «Orthographe et Compréhension verbale » par rapport aux autres niveaux scolaires.

Tableau 9. Résultats des analyses d'homogénéité des variances faites sur les subtests en fonction du niveau scolaire (Seuil de signification $(\mathrm{p}=0,05)$ )

\begin{tabular}{lllll}
\hline & $\begin{array}{l}\text { Statistique } \\
\text { de Levene }\end{array}$ & ddl1 & ddl2 & Sig \\
\hline $\begin{array}{l}\text { Raisonnement } \\
\text { déductif }\end{array}$ & 4,30 & 2,00 & 61,00 & 0,02 \\
\hline $\begin{array}{l}\text { Raisonnement } \\
\text { inductif }\end{array}$ & 0,15 & 2,00 & 61,00 & 0,86 \\
\hline $\begin{array}{l}\text { Problèmes } \\
\text { Attention }\end{array}$ & 5,53 & 2,00 & 61,00 & 0,01 \\
\hline $\begin{array}{l}\text { Orthographe } \\
\text { Compréhension }\end{array}$ & 10,57 & 2,00 & 61,00 & 0,00 \\
\hline verbale & 6,21 & 2,00 & 61,00 & 0,00 \\
\hline
\end{tabular}

Le tableau montre qu'au niveau du test d'hypothèse Levene, il existe une différence significative statistiquement au seuil de signification $(\alpha=0,05)$ entre les sujets selon leur niveau scolaire dans les différents subtests de la batterie NV7 (R1, P, OR, $\mathrm{CV})$ non compris les résultats du subtest « Raisonnement inductif » qui n'indiquent aucune différence significative $(0,15 ; \mathrm{p}=0,86>0,05)$. 
The Journal of Quality in Education (JoQiE) Vol.11, Nº17, May 2021

Tableau 10. Niveau d'adaptation psychosociale des élèves

\section{Effectifs Pourcentage Classement}

(\%)

\begin{tabular}{|c|c|c|c|c|}
\hline \multirow[t]{3}{*}{ Attrait envers l'école } & Faible & 5 & 7,8 & 3 \\
\hline & Moyen & 18 & 28,1 & 2 \\
\hline & Elevé & 41 & 64,1 & 1 \\
\hline \multirow{3}{*}{$\begin{array}{l}\text { Sentiment de compétence en } \\
\text { mathématiques }\end{array}$} & Faible & 2 & 3,1 & 3 \\
\hline & Moyen & 40 & 62,5 & 1 \\
\hline & Elevé & 22 & 34,4 & 2 \\
\hline \multirow{3}{*}{$\begin{array}{l}\text { Sentiment de compétence en } \\
\text { français }\end{array}$} & Faible & 6 & 9,4 & 3 \\
\hline & Moyen & 38 & 59,4 & 1 \\
\hline & Elevé & 20 & 31,3 & 2 \\
\hline \multirow{3}{*}{$\begin{array}{l}\text { Volonté d'apprendre en } \\
\text { mathématiques }\end{array}$} & Faible & 4 & 6,3 & 3 \\
\hline & Moyen & 25 & 39,1 & 2 \\
\hline & Elevé & 35 & 54,7 & 1 \\
\hline \multirow[t]{3}{*}{ Volonté d'apprendre en français } & Faible & 12 & 18,8 & 3 \\
\hline & Moyen & 25 & 39,1 & 2 \\
\hline & Elevé & 27 & 42,2 & 1 \\
\hline
\end{tabular}




\begin{tabular}{lllll}
\hline Orientation vers le travail scolaire & Faible & 1 & 1,6 & 3 \\
\cline { 2 - 4 } & Moyen & 22 & 34,4 & 2 \\
\cline { 2 - 4 } Avantages à décrocher & Elevé & 41 & 64,1 & 1 \\
\hline & Faible & 40 & 62,5 & 1 \\
\hline & Moyen & 22 & 34,4 & 2 \\
\hline Elevé & 2 & 3,1 & 3 \\
\hline Faible & 26 & 40,6 & 2 \\
\hline Moyen & 33 & 51,6 & 1 \\
\hline & Elevé & 5 & 7,8 & 3 \\
\hline Estime de soi & Faible & 19 & 29,7 & 2 \\
\hline Moyen & 43 & 67,2 & 1 \\
\hline & Elevé & 2 & 3,1 & 3 \\
\hline
\end{tabular}

Le tableau ci-dessus indique que le niveau d'adaptation psychosociale varie entre un niveau faible, moyen et élevé, selon les variables relatives à l'adaptation scolaire, la motivation extrinsèque (climat éducatif) et intrinsèque (estime de soi). En effet, on remarque qu'au niveau de l'attrait envers l'école, le sentiment de compétence, la volonté d'apprendre et l'orientation vers le travail scolaire, $28,1 \%$ jusqu'à $62,5 \%$ des sujets atteignent un niveau moyen, $31,3 \%$ jusqu'à $64,1 \%$ des sujets atteignent un niveau élevé, entre $1,6 \%$ et $18,8 \%$ des sujets atteignent un niveau faible. En outre, au niveau de l'avantage à décrocher, $62,5 \%$ des sujets atteignent un niveau faible et $34,4 \%$ des sujets possèdent un niveau moyen. Pour la motivation extrinsèque (climat éducatif), $40,6 \%$ des sujets sont non satisfaits, et $51,6 \%$ satisfaits. De même pour la motivation 
intrinsèque, $29,7 \%$ des sujets possèdent un niveau faible en estime de soi, et $67,2 \%$ des sujets ont un niveau moyen.

Notons que les résultats de l'analyse statistique descriptive ne satisfont pas nos besoins d'identifier les groupes des élèves ayant des difficultés explicites à partir du degré du pouvoir de chaque variable reliée à l'adaptation psychosociale.

\section{Classement des sujets selon les facteurs discriminants}

Tableau 11. Analyse des différences entre groupe

\begin{tabular}{|c|c|c|c|}
\hline Niveau d'adaptation psychosociale & Les variables & $\mathbf{M}$ & ET \\
\hline \multirow[t]{9}{*}{ Non satisfait } & Attrait envers l'école & 5,35 & 1,04 \\
\hline & $\begin{array}{l}\text { Sentiment de compétence en } \\
\text { mathématiques }\end{array}$ & 4,59 & 0,88 \\
\hline & $\begin{array}{l}\text { Sentiment de compétence en } \\
\text { français }\end{array}$ & 4,10 & 0,99 \\
\hline & $\begin{array}{l}\text { Volonté d'apprendre en } \\
\text { mathématiques }\end{array}$ & 4,89 & 1,33 \\
\hline & $\begin{array}{l}\text { Volonté d'apprendre en } \\
\text { français }\end{array}$ & 4,21 & 1,61 \\
\hline & $\begin{array}{l}\text { Orientation vers le travail } \\
\text { scolaire }\end{array}$ & 5,14 & 1,23 \\
\hline & Avantages à décrocher & 5,10 & 0,85 \\
\hline & Climat éducatif & 3,32 & 0,97 \\
\hline & Estime de soi & 3,54 & 0,67 \\
\hline Satisfait & Attrait envers l'école & 5,23 & 1,61 \\
\hline
\end{tabular}


The Journal of Quality in Education (JoQiE) Vol.11, N¹7, May 2021

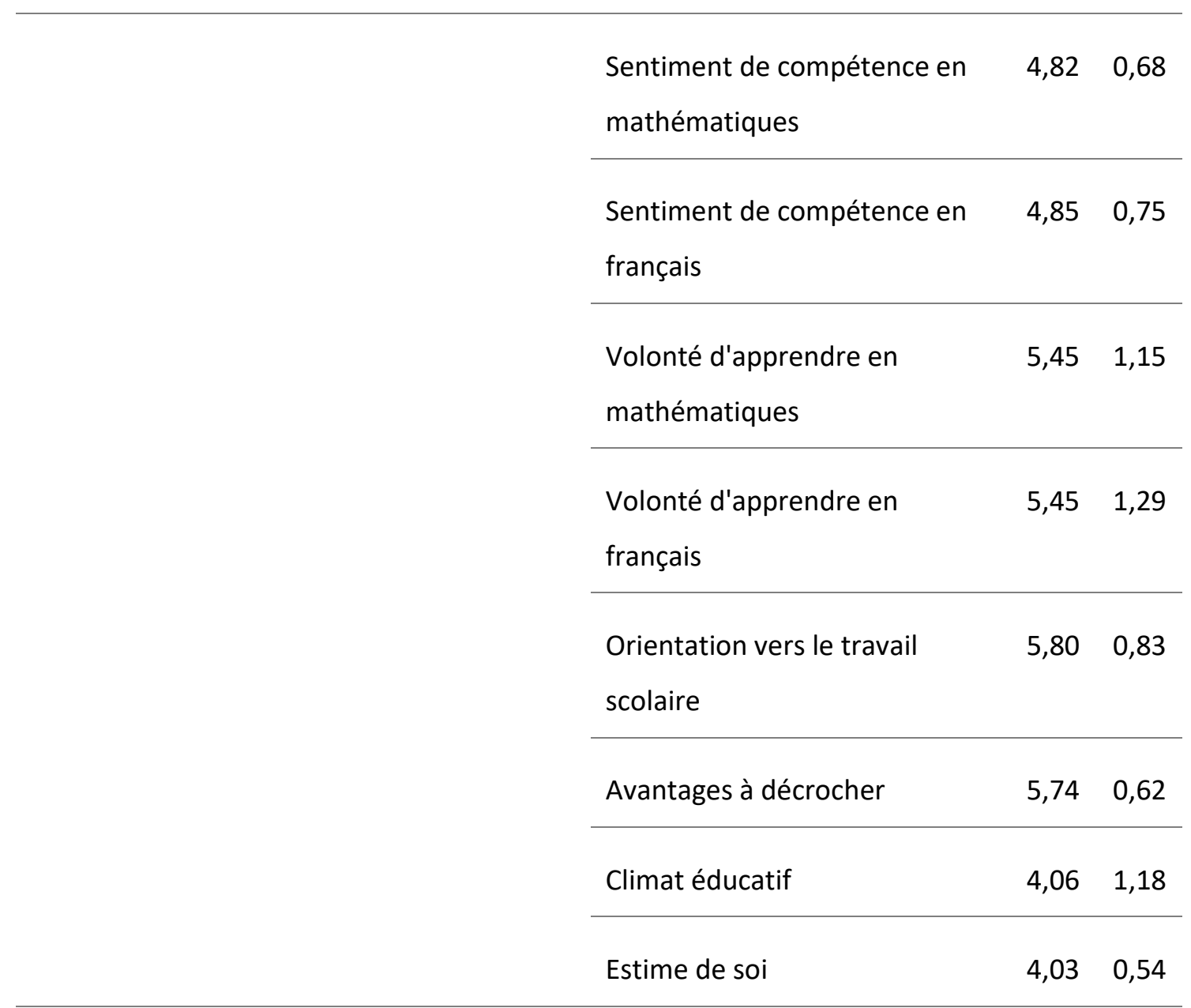

Selon le tableau ci-dessus, on remarque une faible différence au niveau des moyennes et des écart-types. Il s'ensuit qu'il existe un faible pouvoir discriminant des facteurs d'adaptation psychosociale pour la répartition des élèves entre un niveau faible ou moyen, et un autre élevé.

\section{L'adaptation psychosociale et les aptitudes intellectuelles}

Tableau 12. Récapitulatif du modèle

\begin{tabular}{|c|c|c|c|c|c|c|c|c|c|}
\hline \multirow{3}{*}{$\begin{array}{l}\text { Modèl } \\
\text { e }\end{array}$} & \multirow[t]{3}{*}{$\mathbf{R}$} & \multirow[t]{3}{*}{$\mathbf{R}^{2}$} & \multirow{3}{*}{$\begin{array}{l}\mathrm{R}^{2} \\
\text { ajust } \\
\text { é }\end{array}$} & \multirow{3}{*}{$\begin{array}{l}\text { ES de } \\
\text { l'estimatio } \\
n\end{array}$} & \multicolumn{5}{|c|}{ Changement dans les statistiques } \\
\hline & & & & & Variatio & Variatio & ddl & ddl & Sig. \\
\hline & & & & & $n R^{2}$ & $\mathrm{n}$ de $\mathrm{F}$ & 1 & 2 & \\
\hline
\end{tabular}




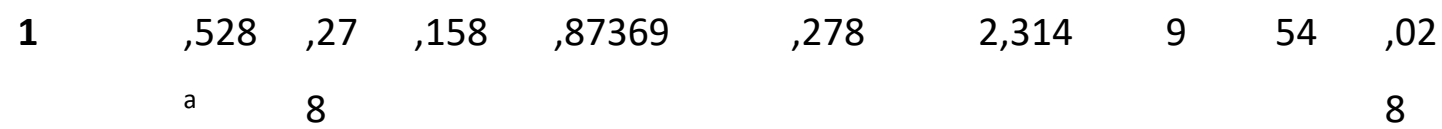

Le tableau ci-dessus montre que la variation de Fisher-Snedecor $(\mathrm{F}=2,314)$ avec un risque de $5 \%$ est significative, d'où le modèle est valide. le coefficient de détermination $\left(\mathrm{R}^{2}=0,278\right)$, signifie que l'adaptation psychosociale (Attrait envers l'école, Sentiment de compétences en mathématiques, Sentiment de compétences en français, Volonté d'apprendre en mathématique, Volonté d'apprendre en français, Orientation vers le travail scolaire, Avantage à décrocher, Climat éducatif et Estime de soi) ne nous permet d'exprimer que $27,8 \%$ de variation du niveau des aptitudes intellectuelles, d'où le modèle restitue $27,8 \%$ de l'information sur le niveau des aptitudes intellectuelles, exprimé dans les variables de l'adaptation psychosociale. Par la suite, $72,2 \%$ de l'information a été perdue.

Tableau 13. Le Coefficient non standardisé ; le coefficient standardisé $\beta$ et le test de Student

\begin{tabular}{|c|c|c|c|c|c|}
\hline \multirow[t]{2}{*}{ Modèle } & \multicolumn{2}{|c|}{$\begin{array}{l}\text { Coefficients non } \\
\text { standardisés }\end{array}$} & \multirow{2}{*}{$\begin{array}{l}\text { Coefficients } \\
\text { standardisés } \\
\text { Bêta }\end{array}$} & \multirow[t]{2}{*}{$\mathrm{t}$} & \multirow[t]{2}{*}{ Sig. } \\
\hline & A & ES & & & \\
\hline (Constante) & 2,434 & 1,353 & & 1,799 & 048 \\
\hline Attrait envers l'école & ,269 & 107 & ,354 & 2,500 & ,016 \\
\hline $\begin{array}{l}\text { Sentiment de compétence en } \\
\text { mathématiques }\end{array}$ & 355 & 181 & ,306 & 1,967 & 044 \\
\hline Sentiment de compétence en français & 209 & 133 & ,214 & 1,572 & 022 \\
\hline Volonté d'apprendre en mathématiques & ,070 & 131 & ,095 &, 534 & ,046 \\
\hline Volonté d'apprendre en français & ,052 & ,082 & ,088 & ,628 & ,032 \\
\hline
\end{tabular}


The Journal of Quality in Education (JoQiE) Vol.11, N¹7, May 2021

\begin{tabular}{|l|l|l|l|l|l|}
\hline Orientation vers le travail scolaire &, 017 &, 125 &, 020 &, 134 &, 894 \\
\hline Avantage à décrocher &,- 175 &, 252 &,- 087 &,- 695 &, 490 \\
\hline Climat éducatif &, 384 &, 224 &, 253 & 1,715 &, 092 \\
\hline Estime de soi &, 214 &, 302 &, 086 &, 711 &, 480 \\
\hline
\end{tabular}

Pour prédire l'influence des variables reliées à l'adaptation psychosociale sur le niveau d'aptitudes intellectuelles, ont été entrés dans le modèle de prédiction : Estime de soi, Volonté d'apprendre en français, Avantage à décrocher, Attrait envers l'école, Sentiment de compétence en mathématiques, Sentiment de compétence en français, Orientation vers le travail scolaire, Climat éducatif, Volonté d'apprendre en mathématiques. Le modèle est significatif, mais tous les liens entre les variables d'adaptation psychosociale et le niveau d'aptitudes intellectuelles ne le sont pas tous.

Les variables suivantes sont prises en compte dans l'équation de régression : Volonté d'apprendre en français $(\mathrm{p}=0,032)$, Attrait envers l'école $(\mathrm{p}=0,016)$, Sentiment de compétence en mathématiques $(\mathrm{p}=0,044)$, Sentiment de compétence en français $(\mathrm{p}=0,022)$, Volonté d'apprendre en mathématiques $(\mathrm{p}=0,046)$, et la constante $(\mathrm{p}=0,048)$. Mais les autres liens ne sont pas pris en compte dans l'équation de régression (le seuil de signification est supérieur à 0,05).

Quand l'attrait envers l'école, le sentiment de compétence et la volonté d'apprendre en mathématique et en français chez les sujets augmentent vers un niveau élevé, la variation du niveau d'aptitudes intellectuelles augmente de 0,269;0,355 ; 0,070;0,209; 0,052 successivement. En somme, selon l'étude de cet échantillon, toutes ces variables portent une influence positive.

Pour prédire l'effet ou l'influence de ces variables précédents sur l'aptitude intellectuelle ; ont été entrés dans le modèle de prédiction : Attrait envers l'école ( $\beta=0$ 
$, 354 ; t=2,500 ; p=0,016<0,05)$, Sentiment de compétence en mathématiques $(\beta=$ $0,306 ; t=1,967 ; p=0,044<0,05)$, Sentiment de compétence en français $(\beta=0,214$; $\mathrm{t}=1,572 ; \mathrm{p}=0,022<0,05)$, Volonté d'apprendre en mathématiques $(\beta=0,095 ; \mathrm{t}=$ $0,534 ; p=0,046<0,05)$, Volonté d'apprendre en français $(\beta=, 088 ; t=, 628 ; p=0,032<$ 0,05). Ce qui montre que l'effet est obtenu avec ses indicateurs pour les variables reliées à l'adaptation psychosociale, alors il est quasiment très léger.

\section{Discussion}

Les résultats indiquent que la plupart des élèves à risque d'échec scolaire présentent un niveau moyen des aptitudes intellectuelles, d'où la présence des acquis scolaires et des potentialités intellectuelles chez ces élèves, surtout dans épreuves suivantes :

- Raisonnement déductif et inductif : les résultats vont quasiment dans le même sens que ceux de l'application NV7 conçue par J.L.Bernaud, P.Priou et R.Simonet $(1991,1992)$ dans le cadre d'un bilan d'orientation destinée aux niveaux $\mathrm{CAP}^{2}$ et $\mathrm{BEP}^{3}$. En effet, la majorité des élèves a une bonne potentialité de progression, d'évolution et d'adaptation à de nouvelles activités. Ceci indique la présence d'une « intelligence fluide », selon la structure des aptitudes cognitives (Carol, 1993), qui leur permet à l'avenir d'éviter la situation d'échec scolaire en achevant leurs études en matières nécessitant la logique, entre autres l'informatique et la programmation.

- Problèmes: Bien que la plupart des problèmes proposés, selon J.L. Bernaud, P. Priou et R. Simonet, semblent simple, les résultats obtenus montrent que certains

\footnotetext{
${ }^{2}$ CAP : Certificat d'Aptitudes Professionnelles-Système éducatif français

${ }^{3}$ BEP : Brevet d'Etudes Professionnelles-Système éducatif français 
élèves présentent des difficultés en mathématiques. La majorité des élèves a un bon potentiel de résolution des problèmes. Le même résultat correspond à l'application NV7 (1990,1991).

- Orthographe, Compréhension verbale et Attention: les résultats obtenus au niveau du présent mémoire et ceux de l'application NV7 vont dans le même sens; le niveau des élèves fluctue entre un niveau faible, moyen et élevé.

Néanmoins, au niveau du subtest «Attention », nos scores sont quasiment plus élevés que ceux de l'application NV7. Etant donné que l'ensemble des résultats des deux premiers subtests montrent que le niveau est globalement moyen, on peut affirmer la présence de «l'intelligence cristallisée », selon la structure des aptitudes cognitives (Carol, 1993). On conclut que les capacités de l'attention dépendent des acquis scolaires, à la différence des propos de Jean-Luc Bernaud et al $(1991,1992)$.

Selon les résultats de l'analyse explicative, aucune différence significative entre les garçons et les filles n'existe au niveau de l'ensemble des épreuves, non compris le subtest «Attention ». En effet, on observe, à la différence de la constatation classique et des études antérieures sur les différences intellectuelles intersexes (voir notamment R.Bonnardel, 1947, 1965 ; P.Priou, I.Parizot et L.Sanchez, 1991), l'absence de supériorité des élèves d'un sexe à ceux d'un autre.

Les résultats montrent également la présence d'une différence significative au niveau des aptitudes intellectuelles entre les élèves d'un niveau scolaire à un autre. D'une part, les élèves en tronc commun professionnel ont un niveau élevé dans les épreuves : Raisonnement déductif, Raisonnement inductif, Problèmes et Attention, chose qui est normale attendu que leur branche nécessite ces capacités. D’autre part, les élèves en $2^{\text {ème }}$ année du baccalauréat présentent un niveau élevé dans les épreuves : 
Orthographe et Compréhension verbale. En effet, les acquis scolaires chez ces élèves sont plus importants que ceux chez des élèves à un niveau inférieur.

Globalement, on peut dire les élèves de l'échantillon présentent des acquis scolaires (intelligence cristallisée), et des potentialités intellectuelles (intelligence fluide). Néanmoins, ils sont insuffisants. On ne peut pas garantir leur réussite. En effet, ils ne sont que des signes de l'apprentissage qui ne prouvent pas qu'un apprentissage s'effectue (Meirieu), la situation d'échec scolaire ne peut pas être évitée.

Les analyses mettent en évidence la présence de traits positifs envers l'école ; les sujets veulent étudier bien que les conditions ne soient pas satisfaites, ces élèves n'auront pas tendance à décrocher, leur estime de soi étant faible jusqu'à moyen.

Le niveau d'adaptation psychosociale selon l'analyse des résultats précédents va dans un sens attendu. Il varie quasiment entre un niveau moyen et élevé quoique la plupart des élèves de l'échantillon présentent des difficultés d'adaptation psychosociale (leur niveau varie entre faible et moyen). En effet, au détriment des conditions, les élèves veulent étudier.

D'après l'analyse discriminative, on conclut que la nature de chaque facteur influençant positivement l'adaptation psychosociale ne contredit pas l'étude faite en ce domaine :

- Sentiment de compétence en français: plus, le sentiment de compétence est élevé, plus l'élève a confiance en soi, plus il est mieux adapté, plus il peut éviter la situation d'échec scolaire, chose qui vérifie ce que Roch Chouinard \& al ont conçu dans leur étude.

- Volonté d'apprendre en français: étant donné que la volonté est la cause de toute action, comme l'affirme Kuhl (1989), et que l'adaptation est tout à fait une 
action, alors, dans ce domaine scolaire, la volonté d'apprendre est un facteur discriminant au niveau de l'adaptation psychosociale.

- Climat éducatif (motivation extrinsèque): D'après Gilles Gendreau, le climat éducatif, faisant partie de l'environnement (conditions mésologiques), influence l'adaptation selon le degré de développement de l'individu. Aussi, Fenouillet admet-il que les conditions de l'environnement expliquent la motivation.

- Estime de soi: C'est parce qu'elle est une catégorie conceptuelle au niveau du motif secondaire, que Fenouillet (2009) la considère comme un facteur expliquant la motivation.

En outre, les résultats de l'analyse de la régression montrent que l'influence de l'ensemble des variables relatives à l'adaptation psychosociale sur le niveau des aptitudes intellectuelles est légère. En effet, bien que la plupart des élèves présentent des difficultés en adaptation psychosociale, ces élèves maintiennent un niveau moyen en aptitudes intellectuelles.

En somme, la situation de l'échec scolaire chez un élève dépend plus particulièrement de l'adaptation de ce dernier que des aptitudes intellectuelles qu'il maintient. Quoique l'élève ne soit pas bien adapté à son environnement, un potentiel intellectuel y réside.

\section{Conclusion}

A partir des données recueillies lors du présent mémoire et à la suite de l'ensemble des analyses faites au cours de l'étude, on retient que 22 élèves n'ont pas de difficultés psychosociales (il se peut que d'autres facteurs influencent l'échec scolaire) au contraire de la majorité au nombre de 42 élèves qui présente des difficultés aussi bien que des capacités intellectuelles. C'est pourquoi, on peut se servir de ces données dans leur 
orientation scolaire vers des branches précises, à savoir celles professionnelles avec leur suivi pédagogique et psychologique en collaboration avec d'autres intervenants.

A partir du modèle calculé, on peut prédire au niveau de l'échantillon les élèves à risque d'échec scolaire ayant des difficultés psychosociales.

La présente étude ne suffit pas à dégager tous les facteurs responsables de l'échec scolaire. Il s'ensuit que d'autres études dont l'échantillon est large interviennent en vue de cerner l'ensemble de ces facteurs vers une remédiation globale.

\section{Bibliographie}

Armano, L. (2004), les tests psychologiques, pour une meilleure connaissance de soi et des autres. Paris, éditions de Vecchi S. A.

Bernaud, J.L. Priou, P \& Simonet, R. NV7: Batterie multifactorielle d'aptitudes, Paris, Ecpa.

Carre, P. \& Fenouillet, F. (2009), Traité de psychologie de la motivation. Paris, Dunod.

Chartrain, X \& Hubert, B. (2000), Prévenir l'échec scolaire. Lyon, Chronique Sociale.

Chartier, P \& Loarer, E. (2008), Evaluer l'intelligence logique, Approche cognitive et dynamique. Paris, Dunod.

Chouinard, R \& al, (2010), Motivation et adaptation psychosociale des élèves du secondaire selon la localisation socioéconomique de leur école. Revue des sciences de l'éducation, vol. 36, $\mathrm{n}^{\circ}$ 2, 2010, p. 321-342. Document téléaccessible à L'URL : $\underline{\text { http://id.erudit.org/iderudit/044480ar. }}$

Francine, B. (1997), L’échec scolaire, Que sais-je, n636, Puf.

Fenouillet, F. (2012), Les théories de la motivation. Paris, Dunod.

François, D. (2014), La démarche d'une recherche en sciences humaines. Québec, De Boeck.

Gimeno José, B. (1984). L'échec scolaire dans l'enseignement primaire: moyens de le combattre, Etudes et enquêtes d'éducation comparée, UNESCO.

Ghewy, P. (2010), Guide pratique de l'analyse de données. Bruxelles, De Boeck.

Imbert, F. (2004), Enfants en souffrance, élève en échec. Paris, ESF éditeur. 
Jocelyne, P, Dominique, T, Gilles Bergeron \& Ginette, L. (2008, 2010), l'évaluation psychoéducative de la personne en difficulté d'adaptation. Québec, Bibliothèque et Archives nationales du Québec.

Lieury, A. \& Fenouillet, F. (1996), Motivation et réussite scolaire. Paris, Dunod.

Laveault, D. \& Gréqoire, J. (2014), Introduction aux théories des tests en psychologie et en sciences de l'éducation. Paris, De Boeck.

Marchand, F. (1997), Evaluation des élèves et conseil de classe. Paris, Editeurs ep1.

Piéron, H. (2003), Vocabulaire de la psychologie. Paris, Puf.

Thiébaut, E \& Bidan-Fortier, C. (2005), NV5R: batterie de tests d'aptitudes cognitives, Paris, Ecpa.

Vianin, P. (2001), contre l'échec scolaire, l'appui pédagogique à l'enfant en difficulté d'apprentissage. Bruxelles, de Boeck.

Viau, R. (1999), la motivation en contexte scolaire, Canada, de Boeck.

Weil-Barais, A. (2001). L'école scolaire: de l'inventaire à la prévention, VEI enjeux, $\mathrm{n}^{\circ}$ 126.

Yanni, E. (2001), comprendre et aider les élèves en échec. Paris, ESF éditeur. 